\title{
Review Article \\ T Lymphocyte Dynamics in Inflammatory Bowel Diseases: Role of the Microbiome
}

\author{
C. B. Larmonier, ${ }^{1}$ K. W. Shehab, ${ }^{1}$ F. K. Ghishan, ${ }^{1}$ and P. R. Kiela ${ }^{1,2}$ \\ ${ }^{1}$ Department of Pediatrics, Steele Children's Research Center, University of Arizona, Tucson, AZ 85724, USA \\ ${ }^{2}$ Department of Immunobiology, University of Arizona, Tucson, AZ 85724, USA
}

Correspondence should be addressed to C. B. Larmonier; larmoncl@email.arizona.edu

Received 3 April 2015; Accepted 28 May 2015

Academic Editor: Tinatin Chikovani

Copyright (C) 2015 C. B. Larmonier et al. This is an open access article distributed under the Creative Commons Attribution License, which permits unrestricted use, distribution, and reproduction in any medium, provided the original work is properly cited.

Humans have coevolved with a complex community of bacterial species also referred to as the microbiome, which reciprocally provides critical contributions to human metabolism and immune system development. Gut microbiome composition differs significantly between individuals depending on host genetics, diet, and environmental factors. A dysregulation of the symbiotic nature of the intestinal host-microbial relationship and an aberrant and persistent immune response are the fundamental processes involved in inflammatory bowel diseases (IBD). Considering the essential role of T cells in IBD and the contributing role of the microbiome in shaping the immune response during the pathogenesis of IBD, this review focuses on the complex relationship, interplay, and communication between the gut microbiome and $\mathrm{T}$ cells, including their differentiation into different subsets of effector or regulatory cells.

\section{Introduction}

Human inflammatory bowel disease (IBD) is a spontaneously relapsing, immunologically mediated disorder of the gastrointestinal tract, characterized by uncontrolled inflammation resulting from inappropriate and persistent activation of the mucosal immune system. Crohn's disease (CD) and ulcerative colitis (UC) represent the two most common forms of the condition, with associated significant morbidity and mortality [1]. Although both environmental factors and genetic predisposition have been implicated in the pathogenesis of IBD [2], the precise causes remain mostly undetermined.

Immune activation within the gut-associated lymphoid tissue (GALT) is essential to counteract potentially harmful pathogens. However, the control of such responses is necessary to avoid an inappropriate immune response against self- or harmless antigens. Disruption of any of the specific immune defense and regulatory mechanisms may lead to the development of chronic intestinal inflammation. Indeed, current evidence suggests that an inappropriate and persistent immune response against the intestinal microbiota plays a pivotal role in the pathogenesis of IBD [3].
The intestinal lamina propria is composed of complex immune cell populations which balance the requirement for physiologic immune tolerance to luminal antigens and the necessity to defend against pathogens. The hallmark of active IBD is an aberrant mucosal infiltration by innate immune cells (primarily neutrophils, macrophages, and dendritic cells) and adaptive immune cells ( $\mathrm{T}$ and $\mathrm{B}$ cells). Effector $\mathrm{CD}^{+}{ }^{+} \mathrm{T}$ cells (Th1, Th2, Th17, and T follicular helper Tfh) are critical in the defense against pathogens, whereas regulatory T cells (nTreg, iTreg; Tr1 and Th3) play a significant role in limiting the expansion and overactivity of $\mathrm{CD} 4^{+}$effector $\mathrm{T}$ cells. IBD seems to be due to either an excessive activation of effector $\mathrm{T}$ cells and/or alteration of $\mathrm{T}$ cell-mediated tolerance mechanisms, the latter through defects in the development of Treg or alteration in their immunosuppressive properties.

The intestinal microbiome has been the subject of intense focus in the last decade, as it is central in both the development of the intestinal immune system and the maintenance of immune tolerance. In particular, Toll-like receptors (TLRs), a family of pattern recognition receptors recognizing conserved commensal bacterial products (including lipopolysaccharide, DNA, and lipoteichoic acid), mediate protection from epithelial injury and play a crucial role in 
the maintenance of intestinal epithelial homeostasis in normal steady state conditions [4]. Additionally, it supplies essential nutrients, modulates energy metabolism, and participates in epithelial cell exfoliation. At least 200-300 different colonic species from 1,800 genera representing between 15,000 and 36,000 individual bacterial species constitute the total microbial load in the intestine [3]. In other terms, the lumen of the distal ileum and the colon contains $10^{14}$ bacteria, whereas the human body is composed of only $10^{12}$ cells. This extremely complex microbiota provides an important source of specific microorganisms, antigens, and ligands that can modulate the activation of the immune system. Indeed, microbial metabolites have a significant impact on disease progression and overall host health and play an important role in the maintenance of gut homeostasis [5].

Dysregulation of the symbiosis between the host intestine and its microbiota and an aberrant and persistent immune response are the fundamental processes involved in inflammatory bowel diseases. Taking into consideration the essential role of $\mathrm{T}$ cells in IBD and the contributing role of the microbiome in the pathogenesis of $\mathrm{IBD}$, this review focuses on the complex relationship and interplay between the gut microbiome and $\mathrm{T}$ cell differentiation in the context of disrupted mucosal homeostasis in IBD.

\section{T Lymphocytes in Immune Tolerance and Inflammation in IBD: General Concepts}

Mucosal innate immune responses are a prerequisite for eliciting adaptive immune responses, as well as for eliciting the adaptive immune responses, which may become the major drivers of tissue injury and establishment of chronic inflammation. The innate components involve a complex network of nonprofessional and professional antigen presenting cells (APC), appropriate sensing of microbialassociated molecular patterns (PAMP) through specialized Toll-like receptors (TLRs) and the nucleotide oligomerization domain- (NOD-) like receptors (NLRs), and microbial control via the production of antimicrobial peptides and mucosal IgA. In recent years, innate lymphoid cells have also become recognized for their important role in mucosal immune homeostasis [6]. More detailed reviews on the innate elements contributing to tolerogenic and pathogenic adaptive immune response have been recently published elsewhere [711].

\footnotetext{
2.1. Role of T Cells in the Maintenance Immune Tolerance in the Gut Mucosa. Mucosa has developed a complex immune system that is capable of mounting an immune response against pathogens, while maintaining the required ignorance or active suppression against nonpathogenic antigens. Immune tolerance is the key mechanism by which intestinal homeostasis is maintained. It refers to the state of active hyporesponsiveness to dietary antigens, commensal enteric bacteria, and orally administrated substances, by a set of mechanisms which evolved to treat external harmless agents to which the immune system is systematically exposed, as an extension of "self."
}

Several types of $\mathrm{T}$ cells exert active immunosuppressive effects in the gastrointestinal tract. "Innate-like" lymphocytes, represented primarily by the TCR $\gamma \delta^{+} \mathrm{T}$ cells, provide signals that enhance barrier function and intercalate between intestinal epithelial cells on the basolateral side of epithelial tight junctions [12]. They respond to epithelial insults by secreting epidermal growth factor (EGF) to promote epithelial repair [13] and by producing proinflammatory cytokines and antimicrobial factors [14] which set the basal mucosal inflammatory tone and limit bacterial translocation.

Past research has shown anticolitogenic properties of $\mathrm{CD}^{+} \mathrm{CD} 28^{-}$cells [15], $\mathrm{CD}^{+} \mathrm{CD} 122^{+}$cells [16], and $\mathrm{CD}^{+} \mathrm{CD}^{-11 c^{+}} \mathrm{T}$ cells [17]. More recently, Liu et al. [18] showed that, in vitro, TGF $\beta$ can induce two distinct populations of $\mathrm{CD}^{+}{ }^{\text {Foxp }} 3^{-}$and $\mathrm{CD}^{+}{ }^{+}$Foxp $^{+}$immunosuppressive T cells. They demonstrated that CD103 expression was obligatory for both populations to be immunosuppressive in vitro and to potently prevent $\mathrm{CD}^{+} \mathrm{T}$ cell-mediated colitis in $\mathrm{Rag} 2^{-/-}$ mice. This finding was consistent with an earlier report by Ho et al. [19], who described a $\mathrm{CD}^{+} \mathrm{CD} 103^{+} \mathrm{T}$ cell population which produced TGF $\beta$, inhibited the proliferation of $\mathrm{CD}^{+}$ in vitro, and attenuated adoptively transferred ileitis in vivo.

The most significant and most extensively studied immunosuppressive lymphocyte populations are $\operatorname{TCR} \alpha \beta$ $\mathrm{CD}^{+}$regulatory Treg cells, characterized by the expression of the Foxp 3 transcription factor. Foxp3 mutations in humans lead to IPEX (immunodysregulation polyendocrinopathy enteropathy X-linked) syndrome, whereas mice lacking Foxp3 develop inflammation-mediated fatal multiorgan failure [20]. Other mutations leading to abnormalities in Treg numbers and function, such as in the WASP, CD25, or IL-10 genes, are also considered risk factors for IBD [21]. Two Foxp $3^{+}$Treg subsets are described: "natural" Treg of thymic origin (nTreg) and "induced" (iTreg), "adaptive," or "peripheral" Treg cells. iTregs develop from naïve T cells in the periphery when activated by transient TCR stimulation or TCR stimulation in the presence of TGF $\beta$ and IL-2. nTregs have the ability to recognize both self-antigens and foreign antigens and play an important role in the maintenance of self-tolerance and prevention of chronic immune stimulation in IBD [22]. After exiting the thymus, nTreg can migrate to the GI tract, where they prevent inappropriate immune responses. nTreg cells act synergistically with iTregs to prevent experimental colitis [23]. iTregs include two main subsets: FoxP $3^{+}$Th 3 cells, secreting predominantly TGF $\beta$, and the Tr1 subset, characterized by lack of Foxp3 expression and high production of IL-10. Both subsets have been implicated in maintaining mucosal homeostasis and in protecting from experimental colitis [24-26]. More detailed reviews on the role of Tregs, their lineages, and respective functions in the intestinal mucosal homeostasis have been recently published $[21,27]$.

\subsection{Types and Roles of Effector T Cells in the IBD-Associated} Pathogenic Immune Responses. $\mathrm{CD}^{+}{ }^{-} \mathrm{T}$ lymphocytes are believed to play a key role in the pathogenesis of human IBD, as evidenced by their influx into the inflamed mucosa, the effectiveness of depleting anti-CD4 antibody therapies [28], or the suppression of Crohn's disease symptoms observed 
in individuals with concomitant HIV infection [29]. The essential role of $\mathrm{CD}^{+} \mathrm{T}$ cells has been demonstrated in several animal models of experimental colitis, most notably in the adoptive naïve $\mathrm{T}$ cell transfer into a lymphopenic host [30]. Furthermore, this model of colitis is dependent upon the presence of bacteria, as it does not occur under germ-free conditions [31].

Traditionally, it was believed that CD was characterized predominantly by Th1 T cells secreting IFN $\gamma$, which were induced via the IL-12/IL-27-dependent differentiation pathway. In contrast, UC was thought to be mediated by Th2 $\mathrm{T}$ cells, associated with IL-4, IL-5, and IL-13 production. However, in recent years, the Th1/Th2 paradigm has been considerably revised. The role of Thl7 cells in the adaptive immune response in IBD has been described. The Th17 subset differentiates from naïve $\mathrm{T}$ cells in the presence of IL-6 and TGF $\beta$ and secretes IL-17 and IL-22. Primarily secreted by activated dendritic cells (DC) and macrophages, IL-23 is important in the maintenance of the Thl7 cell lineage. Elevated IL-17 and IL-22 levels have been found in the gut mucosa of patients with UC and CD [32]. Importantly, a simplistic model of any one lineage driving the disease pathogenesis is difficult to prove. As an example, an enrichment of IFN $\gamma^{+} \mathrm{IL}-17^{+}$coproducing $\mathrm{CD} 4^{+} \mathrm{T}$ cells with a proinflammatory phenotype, previously ascribed to $\mathrm{CD}$ pathogenesis, can also be found in actively inflamed mucosal lesions from UC patients [33]. Moreover, involvement of different Teff cells can change during the course of disease as exemplified by the SAMP1/YitFc mouse, a model of spontaneous CDlike ileitis. In these mice, ileitis initiation is mediated by a Th1 response with IFN $\gamma$ and TNF $\alpha$ production, but the establishment of chronic inflammation is dependent upon a Th2 response, with secretion of IL-5 and IL-13 [34]. Another layer of complexity is added when $\mathrm{T}$ cell plasticity is considered, as exemplified by the Foxp $3^{+}$IL-17-producing CD $4^{+} \mathrm{T}$ cells enriched in active IBD $[35,36]$ or the aforementioned IFN $\gamma^{+}$IL- $17^{+} \mathrm{CD}^{+}{ }^{+} \mathrm{T}$ cells. IL-21-producing follicular helper $\mathrm{T}$ cells (Tfh) have also been recently characterized in IBD patients, although IL-21 production can also be acquired by IFN $\gamma^{+}$Th1 T cells [37].

The pathogenic role of autoreactive $\mathrm{CD} 8^{+} \mathrm{T}$ cells in IBD has been demonstrated in murine studies and implied by observations in IBD patients. Low avidity autoreactive $\mathrm{CD} 8^{+}$ $T$ cells can escape both central and peripheral tolerance and may trigger autoimmune reactions to a microbial mimic of self-antigen [38]. Funderburg et al. [39] recently documented elevated numbers of activated $\mathrm{CD} 8^{+} \mathrm{T}$ cells in IBD patients. Animal studies support the role of pathogenic MHC class I-restricted $\mathrm{CD}^{+}{ }^{+} \mathrm{T}$ cells in response to self- or exogenous antigens in the intestinal mucosa [40-42].

\section{Microbiome and T Cells}

A metagenomic catalog of the human gut microbial gene catalog, using next generation sequencing approaches, was recently published $[43,44]$. Ninety-nine percent of the microbial genes were bacterial. Approximately 160 species were identified, largely shared amongst individuals [43]. Gut commensal bacteria play critical roles in making nutrients accessible to the host, in the development of the immune system, and in the host's metabolism. In healthy individuals, the intestinal microbiome is dominated by four major bacterial phyla: Bacteroidetes, Firmicutes, Proteobacteria, and Actinobacteria. Reduced phylogenetic diversity has been described in IBD patients. Indeed, a relative decrease in Firmicutes (including Clostridia groups IV and XIVa) and an increase increase in Bacteroidetes, Proteobacteria, and Actinobacteria have been observed consistently among IBD patients [45-47]. The pathogenic role of commensal bacteria in IBD is implicated by the fact that most germ-free (GF) colitis-susceptible rodents have no intestinal inflammation or immune activation but rapidly develop pathogenic immune responses and disease after colonization with specific pathogen-free enteric bacteria [48]. Moreover, GF mice have relatively underdeveloped GALT [49]. Therefore, specific members of the gut microbiota were postulated to orientate host-specific $\mathrm{T}$ cell responses and modulate $\mathrm{T}$ cell differentiation.

3.1. Effect of the Microbiome on Effector T Cells. The importance of the microbiome relative to $\mathrm{T}$ cells is evidenced by the fact that the overall numbers of both $\mathrm{CD}^{+}$and $\mathrm{CD} 8^{+}$ $\mathrm{T}$ cells are decreased in germ-free mice but are restored after recolonization [50] and that gut immune maturation is dependent upon colonization with host-specific microbiota [51]. However, upon infection with the intestinal parasite Toxoplasma gondii, $\mathrm{CD} 4^{+} \mathrm{T}$ cells respond against the pathogen as well as the translocating commensal bacteria, demonstrating the ability of the immune response to override tolerance to commensals [52]. The key role of the microbiota on $\mathrm{T}$ cell plasticity has been illustrated by the recent isolation of $\mathrm{T}$ cell clones specific for bacteria such as the Enterobacteriaceae, Bacteroides, and Bifidobacterium [53]. Functional relevance of those bacteria specific $\mathrm{T}$ cell clones was demonstrated by the transfer of clostridial flagellin-specific $\mathrm{CD} 4^{+}$effector $\mathrm{T}$ cells into Toxoplasma-infected mice, which are able to induce inflammation in the colon [54].

Bacteroides fragilis, a gram-negative anaerobic bacteria and its capsular polysaccharide A (PSA) have been described to modify the Th1/Th2 $\mathrm{T}$ cell balance in mice. This mechanism was shown to be dependent upon the PSA zwitterionic charge motif, which allows for its processing by dendritic cells. PSA signals through TLR2 and initiates the production of cytokines modulating Th1/Th2 balance (activation of NF- $\kappa \mathrm{B}$, regulation of TNF $\alpha$, IL-12 production). PSA function in the regulatory arm of the adaptive immune system is described in more detail below [55].

In the absence of commensal bacteria, germ-free or antibiotic-treated mice have significantly fewer Thl7 cells than under normal conditions. It is well accepted that commensal bacteria prime Th17 differentiation [56]. In particular, adenosine $5^{\prime}$-triphosphate (ATP) derived from commensal bacteria has been shown to activate $\mathrm{CD} 11 \mathrm{c}^{\text {low }} \mathrm{CD} 70^{\text {high }}$ cells in the lamina propria, driving Th17 cells differentiation [57]. In addition, certain commensal bacteria can modulate the production of IL-17 by gut resident $\gamma \delta \mathrm{T}$ cells in the small intestine. Duan and colleagues have demonstrated that commensal bacteria participate in the expansion and 
maintenance of $\mathrm{CD} 2 \mathrm{~L}^{-}$IL- $1 \mathrm{R}^{+} \gamma \delta \mathrm{T}$ cells and that IL-1 signaling is necessary for the secretion of IL-17 by $\gamma \delta \mathrm{T}$ cells, highlighting the essential role of endogenous flora [58]. In addition, some commensal bacteria can modulate the production of IL-17 by gut resident $\gamma \delta$ T cells in the small intestine [58]. One microbial group collectively termed Segmented Filamentous Bacteria (SFB), a nonculturable group of Clostridia-related organisms, have been shown to play a role in the differentiation of the Th17 $\mathrm{T}$ cell lineage as well as IL-22-producing $\mathrm{CD}^{+}{ }^{+} \mathrm{T}$ cells in the small intestine and the colon $[59,60]$. The specific role of SFB in inducing Th17 responses remains unclear, although it is believed that SFB can stimulate the production of the early inflammatory marker serum amyloid A (SAA), which in turn stimulates a specific Th17 response in vitro [60]. Moreover, colonizing germ-free mice with SFB partially restores the $\alpha \beta$ TCR IEL population, highlighting the importance of SFB on gut immune maturation [51]. SFB mediate protection against enteric infection by Citrobacter rodentium [59, 60]. Taken together, these findings suggest that SFB play a dual role in the maintenance of gut homeostasis. SFB are present in increased numbers in UC patients, suggesting their potential importance in human disease [61]. Lastly, thymic stromal lymphopoietin (TSLP), which is constitutively expressed in the intestine in the presence of intestinal bacteria, can play a role in the expansion of Th17 cells and also promotes Helios ${ }^{-}$Foxp $^{+}$regulatory T cells [62].

GF animals harbor similar numbers of "innate-like" TCR $\gamma \delta$ T cells compared to conventionally housed mice, yet under GF conditions these cells have a diminished ability to enhance mucosal repair [14]. TCR $\gamma \delta \mathrm{T}$ cells are able to control opportunistic translocation of commensal bacteria following intestinal epithelial injury and can be induced by commensal bacteria to produce antimicrobial peptides (Reg $3 \gamma$ by IEL and LPL TCR $\gamma \delta$ T cells). The cumulative effect is a direct contribution to modulating the composition of the microbiota as well as containing invading enteric pathogens $[14,58]$.

Commensal bacteria also modulate the balance between regulatory and effector $\mathrm{T}$ cells via pattern recognition receptors, including TLRs. For instance, $T l r 9^{-/-}$mice have an increased frequency of Treg and reduced IFN $\gamma$ - and IL-17producing Teff cells in the small intestine. This phenotype is reversed with administration of TLR9 ligands, indicating that commensal DNA or CpG have the ability to guide the mucosal responses to infection by a TLR9-dependent mechanism [63]. The role of TLR5 is also prominent, albeit complex. TLR5 stimulation promotes Teff cells while opposing Treg generation in vitro. Tlr $5^{-/-}$mice develop spontaneous colitis, despite an increased number of Foxp $3^{+}$Tregs compared to WT littermates. TLR5-deficient mice have transiently increased levels of Proteobacteria, especially Enterobacteriaceae species (including E. coli), observed in close proximity to the gut epithelium. This has been attributed to a defect in innate sensing, which leads to unstable gut microbiota and low-grade inflammation, which may represent drivers for chronic colitis [64]. In the adaptive branch, dendritic cell stimulation by flagellin/TLR5 leads to an increase in IL-23, which further promotes the Th17 effector cell pathway to the detriment of Foxp $3^{+}$Treg $[65,66]$. Moreover, certain flagellins have been postulated to be dominant antigens in the pathogenesis of CD. C3H/HeJBir mice, a substrain which develops spontaneous colitis, were used to identify a family of related novel flagellins, which activate TLR5, as a class of immunodominant antigens [67]. These flagellin clones were closely related to flagellins from Butyrivibrio, Roseburia, Thermotoga, and Clostridium within the Clostridium subphylum cluster XIVa. Antibodies to Cbirl, one of the commensalderived flagellins, were also detected in $M d r 1^{-/-}$and $\mathrm{Il-10^{-/- }}$ mice and in CD patients, while Cbirl-specific $\mathrm{CD}^{+}{ }^{+} \mathrm{T}$ cells induced severe colitis when adoptively transferred into naive SCID mice [67].

\subsection{Effect of the Microbiome on Regulatory T Cells. Although} regulatory $\mathrm{T}$ cells play a critical role in the maintenance of gut homeostasis, commensal bacteria may not be required for the generation of Tregs in the small intestine. Indeed, Treg frequency and immunosuppressive function are the same in the small intestine of germ-free mice compared to SPF mice $[63,68]$. However, colonic Treg are significantly reduced in germ-free mice, suggesting a critical role of the colonic microbiota in Treg generation [69].

The Ikaros-family transcription factor Helios, a putative marker for thymic nTregs, has been used to study the impact of nTreg in the colon and the role of the microbiota in differentiation of naïve peripheral $\mathrm{T}$ cells into colonic Tregs. In GF mice, colonic Tregs are Helios ${ }^{\text {high }}$, in contrast to conventional mice, in which colonic Tregs are mostly Helios $^{\text {low }}$. This suggested that colonic Tregs are converted from $\mathrm{CD}^{+} \mathrm{T}$ cells in the periphery [70]. In conventional mice, colonic Tregs are therefore primarily differentiated outside the thymus in the gut in response to foreign antigens. These findings were replicated using another marker for thymic-derived Treg, neuropilin 1 [70].

The ways by which bacterial-derived molecules coordinate peripherally generated Treg are illustrated in work done with Clostridia and Bacteroides fragilis, as both increase the frequency and stimulate the function of colonic Treg. Similar to what has been observed with effector $\mathrm{T}$ cells, colonic commensal luminal antigens can shape the intestinal Treg pool via the selection of specific TCRs and promote the efficient generation of peripheral Treg tolerant of the commensal microbiota [70].

One of the best examples of Treg modulation by the microbiome comes from studies on Bacteroides fragilis. Capsular lipopolysaccharide A (PSA) is primarily responsible for the immunostimulatory and immunoregulatory potential of $B$. fragilis. PSA has been shown to signal through the TLR2/MyD88 pathway in dendritic cells and also directly on $\mathrm{CD}^{+}$T cells. B. fragilis promotes IL-10-producing Foxp $3^{+}$ Treg $[71,72]$. As mentioned above, GF mice have a general defect in colonic $\mathrm{CD}^{+}{ }^{+} \mathrm{T}$ cell development; $B$. fragilis is able to correct these deficiencies [71]. Treg induction by B. fragilis is driven by PSA and is dependent upon TLR2 signaling to induce IL-10 production (increased mRNA for IL-10, TGF $\beta 2$, GITR, ICOS, CTLA4, and Ebi3; IL-27 $\beta$ ), 
an effect that is restricted to the Foxp $3^{+}$Treg population. These observations constitute some of the seminal evidence describing the specific ways an individual constituent of the microbiota can control inflammatory responses.

More recently, the role of Clostridia, primarily clusters IV (Clostridium leptum) and XIVa (Clostridium coccoides) in intestinal homeostasis, has been investigated. These clusters were found to be responsible for the accumulation of $\mathrm{CD}^{+}{ }^{+} \mathrm{CD} 25^{+} \mathrm{Foxp}^{+}{ }^{+}$Treg (Helios ${ }^{-}$, CTLA4, and IL-10 ${ }^{\text {high }}$ ) in the colonic lamina propria [73] and suggested that Clostridia are potent inducers of peripheral Treg differentiation and proliferation, presumably in the gut. Reconstitution of germfree mice with a mix of 46 Clostridial strains induced the accumulation of a significant number of Tregs to levels similar to those of SPF mice in the cecum and the colon (but not in the small intestine). The specific effect of Clostridium species on Treg was further emphasized by the fact that colonization of germ-free mice by 16 Bacteroides species, SFB, and 3 Lactobacillus specieshad a minimal effect on colonic Treg accumulation [73]. In addition, early colonization of SPF mice with Clostridia generated a Clostridia-rich environment, which was protective from experimental colitis. Similarly, the colonization of GF mice with 17 Clostridia species from human fecal microbiota was also capable of Foxp $3^{+}$ Treg induction and inhibition of colitis in TNBS and adoptive $\mathrm{T}$ cell transfer models [74]. Tregs from Clostridia-treated colitic mice were competent to inhibit OT-I TCR CD8 ${ }^{+} \mathrm{T}$ cell antigen specific proliferation in vitro. This inhibition was even greater with the addition of autoclaved cecal contents from Clostridia-treated mice. Clostridia-mediated Treg generation appears, therefore, to be antigen-specific [74].

The protective effect of Clostridia species may be mediated to a large extent by SCFA (short chain fatty acids; acetic acid, propionic acid, and butyric acid). SCFA are believed to support the expansion of the existing pool of colonic Tregs, and butyrate alone has been demonstrated to induce Treg differentiation both in vivo and in vitro, enhancing histone acetylation in the promoter and conserved regions of the Foxp3 locus $[75,76]$. In another study, Singh et al. [77] showed that, in colonic antigen presenting cells, signaling through butyrate and the niacin receptor Gpr109a, coded by Niacrl gene, permits differentiation of Treg cells and IL-10-producing T cells. Gpr109a was required for the expression of IL-18, and $\mathrm{Niacr}^{-1-}$ mice showed enhanced susceptibility to colitis and colon cancer. Moreover, depletion of gut microbiota or dietary fiber increased the risk for colitis and cancer, which was suppressed by niacin in a Gpr109adependent manner [77].

The probiotic VSL\#3 (comprised of Bifidobacterium breve, Bifidobacterium longum subsp. infantis, Lactobacillus acidophilus, Lactobacillus plantarum, Lactobacillus paracasei, Lactobacillus bulgaricus, and Streptococcus thermophilus) reduced the severity of experimental colitis, which correlated with diminished effector T cell cytokine levels $[78,79]$ and with increased number of TGF $\beta$ - and IL-10-producing Tregs [80]. In addition, Bifidobacterium longum subsp. infantis has been shown to prevent Salmonella typhimurium-driven inflammation by increasing Treg numbers [81].
Recently, another gut commensal bacteria, Faecalibacterium prausnitzii, which typically is found in reduced abundance in IBD patients [82], was found to induce $\mathrm{CD} 4^{+} \mathrm{CD} 25^{+}$Foxp $^{+}$Treg [83].

\section{T Cells Can Also Shape the Gut Microbiome}

Although less well-studied, there is emerging evidence which demonstrates the reciprocal relationship between $\mathrm{T}$ cells and their ability to shape the composition of the gut microbiota. Indeed, defects in competent adaptive immunity can modulate the response to the microbiota and has been linked to substantial changes in microbial composition. In a recent study, Zhang et al. [84] studied the microbiome composition in Rag1 deficient mice and WT mice. This report demonstrated that the phylogenetic composition of the microbiota was different in $\mathrm{Ragl}^{-/-}$before and after weaning. In particular, Akkermansia muciniphila was enriched in immunodeficient mice. The lack of $\mathrm{B}$ and $\mathrm{T}$ cells also correlated with a decrease in Lactobacillales and Enterobacteriales in the pre-weaning stage and increased Verrucomicrobiales in the pre- and postweaning periods. Furthermore, $\mathrm{Ragl}^{-1-}$ mice showed a significant increase in microbial diversity with age compared to WT, pointing out the role of adaptive immune system pressure in modulating gut microbiota composition [84]. SFB have also been shown to be more abundant in

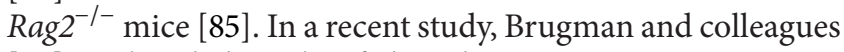
[86] analyzed the role of the adaptive immune system on $\mathrm{Ragl}^{-1-}$ deficient zebrafish and showed that Vibrio species abundance increased. They also confirmed that adoptive $\mathrm{T}$ cell transfer in Ragl deficient zebrafish suppressed the overgrowth of Vibrio. The study was completed by an elegant $e x$ vivo experiment where exposure of intestinal $\mathrm{T}$ lymphocytes to $\mathrm{Ragl}^{-/-}$derived microbiota increased IFN $\gamma$ expression.

TRUC mice $\left(\right.$ Tbet $^{-1-} \times \mathrm{Rag}^{-{ }^{--}}$) display a defect in competent adaptive immune responses and develop spontaneous colitis, which can be controlled by Treg or antibiotic treatment. Klebsiella pneumoniae and Proteus mirabilis were found to be present in abundance in TRUC mice, linking a defect in the response to the microbiota to changes in the microbial composition [87]. Kawamoto et al. [88] described that Tregs are necessary for the maintenance of microbial diversity, including a promoting effect on the abundance of nonpathogenic Clostridia. These and other studies described in this review point to the elaborate and interdependent relationship between $\mathrm{T}$ cells, Treg in particular, and the gut microbiome.

\section{Conclusions}

The studies outlined in this review provide strong evidence that the gut microbiota and $\mathrm{CD} 4^{+} \mathrm{T}$ cell relationship is dynamic and interdependent, with each affecting the composition/function of the other (Figure 1). The microbial composition of the host at different sites (small intestine or colon) plays a major role in the balance of Treg/Teff in the gut, whether this is driven by the production of microbial metabolites (e.g., SCFA), by colonization by SFB or other 


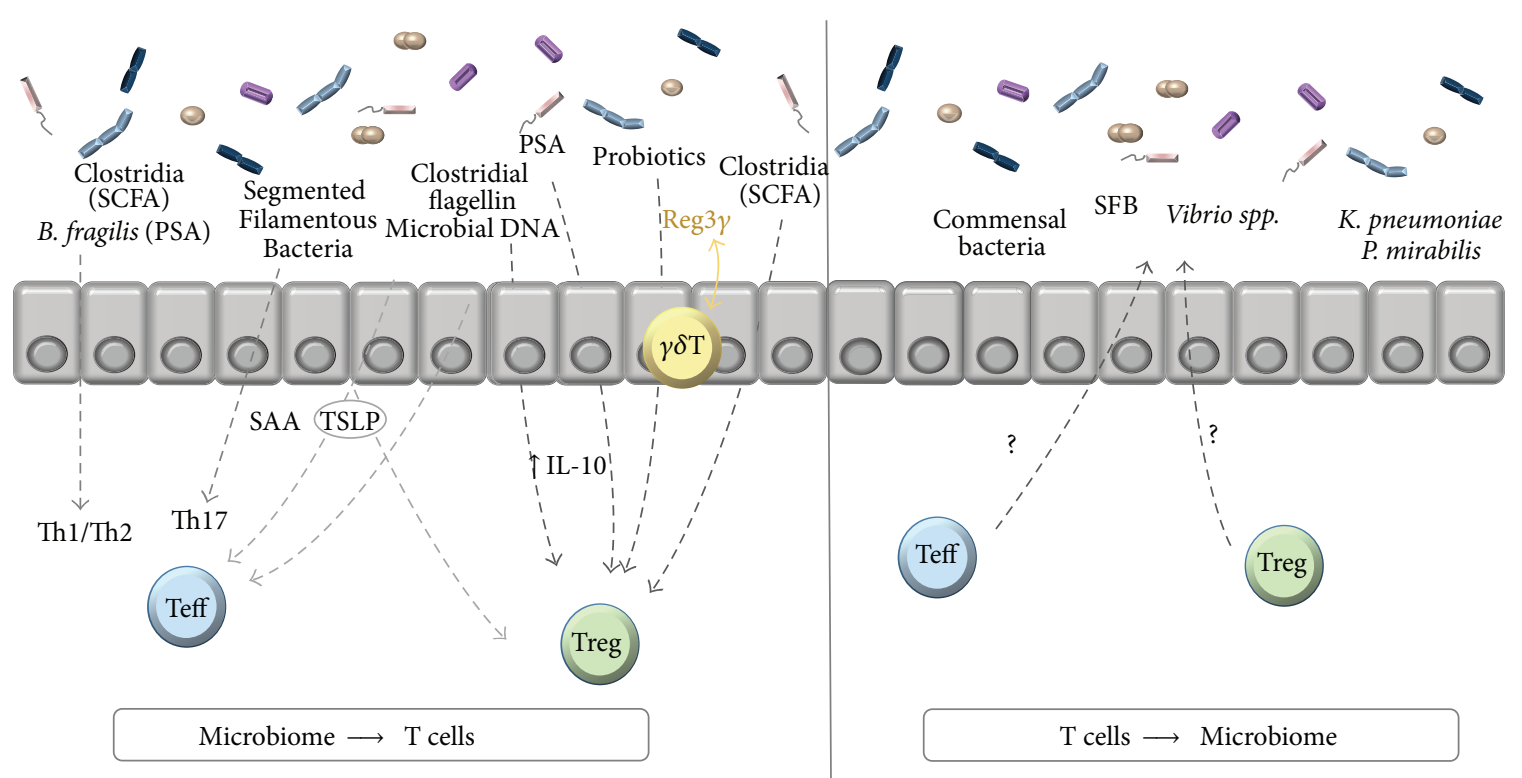

FIGURE 1: Schematic depiction of the major points emphasized in the review.

organisms, or through innate signaling via TLRs (mostly TLR5 and TLR9). In this review, we focused on the role of the microbiota on effector and regulatory $\mathrm{T}$ cells. The role of antecedent stimulation of the innate immune system as a prerequisite for $\mathrm{T}$ cell activation and dysregulation in colitis (the so-called "two-hit" model) is discussed elsewhere [89] and was beyond the scope of this review.

As our knowledge of the nuanced relationship between the microbiota and the adaptive immune system develops, it is tempting to speculate about the therapeutic potential of manipulating the microbiota to influence $\mathrm{T}$ cell responses and vice versa in IBD. Therapeutic manipulation of the microbiome for various other indications, including fecal transplantation for Clostridium difficile colitis and administration of probiotics, has been the subject of significant recent attention but has not yet been translated to the management of IBD [90]. Considering the mutualistic coevolution of the gut microbiome and host immune maturation, investigating this complex relationship has the potential to positively impact patients with IBD. Additional studies that translate the findings described in this review into clinical therapeutics are needed and highly anticipated.

\section{Conflict of Interests}

The authors declare no conflict of interests to disclose.

\section{Authors' Contribution}

C. B. Larmonier and K. W. Shehab shared authorship.

\section{Funding}

This work was funded by NIDDK 1K01DK099268-01 (to C. B. Larmonier) and 5R01 DK041274 (to P. R. Kiela and F. K. Ghishan).

\section{References}

[1] Z. Kassam, S. Belga, I. Roifman et al., "Inflammatory bowel disease cause-specific mortality: a primer for clinicians," Inflammatory Bowel Diseases, vol. 20, no. 12, pp. 2483-2492, 2014.

[2] R. J. Xavier and D. K. Podolsky, "Unravelling the pathogenesis of inflammatory bowel disease," Nature, vol. 448, no. 7152, pp. 427-434, 2007.

[3] R. B. Sartor, "Microbial influences in inflammatory bowel diseases," Gastroenterology, vol. 134, no. 2, pp. 577-594, 2008.

[4] S. Rakoff-Nahoum, J. Paglino, F. Eslami-Varzaneh, S. Edberg, and R. Medzhitov, "Recognition of commensal microflora by toll-like receptors is required for intestinal homeostasis," Cell, vol. 118, no. 2, pp. 229-241, 2004.

[5] G. Sharon, N. Garg, J. Debelius, R. Knight, P. C. Dorrestein, and S. K. Mazmanian, "Specialized metabolites from the microbiome in health and disease," Cell Metabolism, vol. 20, no. 5, pp. 719-730, 2014.

[6] G. F. Sonnenberg, "Regulation of intestinal health and disease by innate lymphoid cells," International Immunology, vol. 26, no. 9, pp. 501-507, 2014.

[7] P. P. Elia, Y. F. Tolentino, C. Bernardazzi, and H. S. de Souza, "The role of innate immunity receptors in the pathogenesis of inflammatory bowel disease," Mediators of Inflammation, vol. 2015, Article ID 936193, 10 pages, 2015.

[8] M. Rescigno, "Dendritic cell-epithelial cell crosstalk in the gut," Immunological Reviews, vol. 260, no. 1, pp. 118-128, 2014.

[9] A. M. Mowat and W. W. Agace, "Regional specialization within the intestinal immune system," Nature Reviews Immunology, vol. 14, no. 10, pp. 667-685, 2014.

[10] M. Z. Cader and A. Kaser, "Recent advances in inflammatory bowel disease: mucosal immune cells in intestinal inflammation," Gut, vol. 62, no. 11, pp. 1653-1664, 2013.

[11] J. M. Davies and M. T. Abreu, "The innate immune system and inflammatory bowel disease," Scandinavian Journal of Gastroenterology, vol. 50, no. 1, pp. 24-33, 2015. 
[12] J. E. Dalton, S. M. Cruickshank, C. E. Egan et al., "Intraepithelial gammadelta+ lymphocytes maintain the integrity of intestinal epithelial tight junctions in response to infection," Gastroenterology, vol. 131, no. 3, pp. 818-829, 2006.

[13] Y. Chen, K. Chou, E. Fuchs, W. L. Havran, and R. Boismenu, "Protection of the intestinal mucosa by intraepithelial $\gamma \delta \mathrm{T}$ cells," Proceedings of the National Academy of Sciences of the United States of America, vol. 99, no. 22, pp. 14338-14343, 2002.

[14] A. S. Ismail, C. L. Behrendt, and L. V. Hooper, "Reciprocal interactions between commensal bacteria and $\gamma \delta$ intraepithelial lymphocytes during mucosal injury," The Journal of Immunology, vol. 182, no. 5, pp. 3047-3054, 2009.

[15] I. Menager-Marcq, C. Pomie, P. Romagnoli, and J. P. M. van Meerwijk, "CD8 ${ }^{+} \mathrm{CD} 28^{-}$regulatory $\mathrm{T}$ lymphocytes prevent experimental inflammatory bowel disease in mice," Gastroenterology, vol. 131, no. 6, pp. 1775-1785, 2006.

[16] A. T. Endharti, Y. Okuno, Z. Shi et al., " $\mathrm{CD} 8^{+} \mathrm{CD} 122^{+}$regulatory $\mathrm{T}$ cells (Tregs) and $\mathrm{CD} 4^{+}$Tregs cooperatively prevent and cure $\mathrm{CD}^{+}{ }^{+}$cell-induced colitis," Journal of Immunology, vol. 186, no. 1, pp. 41-52, 2011.

[17] D. Fujiwara, L. Chen, B. Wei, and J. Braun, "Small intestine $\mathrm{CD}_{11 c^{+}} \mathrm{CD}^{+} \mathrm{T}$ cells suppress $\mathrm{CD} 4^{+} \mathrm{T}$ cell-induced immune colitis," American Journal of Physiology: Gastrointestinal and Liver Physiology, vol. 300, no. 6, pp. G939-G947, 2011.

[18] Y. Liu, Q. Lan, L. Lu et al., "Phenotypic and functional characteristic of a newly identified $\mathrm{CD} 8^{+} \mathrm{Foxp}^{-} \mathrm{CD}^{-} 103^{+}$regulatory T cells," Journal of Molecular Cell Biology, vol. 6, no. 1, pp. 81-92, 2014.

[19] J. Ho, C. C. Kurtz, M. Naganuma, P. B. Ernst, F. Cominelli, and J. Rivera-Nieves, "A CD8+/CD103high T cell subset regulates TNF-mediated chronic murine ileitis," The Journal of Immunology, vol. 180, no. 4, pp. 2573-2580, 2008.

[20] J. D. Fontenot, M. A. Gavin, and A. Y. Rudensky, "Foxp3 programs the development and function of $\mathrm{CD} 4{ }^{+} \mathrm{CD} 25^{+}$regulatory T cells," Nature Immunology, vol. 4, no. 4, pp. 330-336, 2003.

[21] C. G. Mayne and C. B. Williams, "Induced and natural regulatory T cells in the development of inflammatory bowel disease," Inflammatory Bowel Diseases, vol. 19, no. 8, pp. 1772-1778, 2013.

[22] S. Sakaguchi, "Naturally arising CD4+ regulatory $\mathrm{T}$ cells for immunologic self-tolerance and negative control of immune responses," Annual Review of Immunology, vol. 22, pp. 531-562, 2004.

[23] D. Haribhai, J. B. Williams, S. Jia et al., "A requisite role for induced regulatory $\mathrm{T}$ cells in tolerance based on expanding antigen receptor diversity," Immunity, vol. 35, no. 1, pp. 109-122, 2011.

[24] C. L. Maynard, L. E. Harrington, K. M. Janowski et al., "Regulatory $\mathrm{T}$ cells expressing interleukin 10 develop from Foxp $^{+}$and Foxp $3^{-}$precursor cells in the absence of interleukin 10," Nature Immunology, vol. 8, no. 9, pp. 931-941, 2007.

[25] H. Groux, A. O'Garra, M. Bigler et al., "A CD4 ${ }^{+}$T-cell subset inhibits antigen-specific T-cell responses and prevents colitis," Nature, vol. 389, no. 6652, pp. 737-742, 1997.

[26] M. C. Fantini, C. Becker, I. Tubbe et al., "Transforming growth factor $\beta$ induced FoxP3+ regulatory $\mathrm{T}$ cells suppress Th1 mediated experimental colitis," Gut, vol. 55, no. 5, pp. 671-680, 2006.

[27] J. Bollrath and F. M. Powrie, "Controlling the frontier: regulatory T-cells and intestinal homeostasis," Seminars in Immunology, vol. 25, no. 5, pp. 352-357, 2013.
[28] J. Emmrich, M. Seyfarth, W. E. Fleig, and F. Emmrich, “Treatment of inflammatory bowel disease with anti-CD4 monoclonal antibody," The Lancet, vol. 338, no. 8766, pp. 570-571, 1991.

[29] B. Greenwald and S. P. James, "Long-term HIV infection with Crohn's disease," American Journal of Gastroenterology, vol. 90, no. 1, pp. 167-168, 1995.

[30] D. V. Ostanin, J. Bao, I. Koboziev et al., "T cell transfer model of chronic colitis: concepts, considerations, and tricks of the trade," The American Journal of Physiology-Gastrointestinal and Liver Physiology, vol. 296, no. 2, pp. G135-G146, 2009.

[31] A. Izcue, J. L. Coombes, and F. Powrie, "Regulatory T cells suppress systemic and mucosal immune activation to control intestinal inflammation," Immunological Reviews, vol. 212, pp. 256-271, 2006.

[32] S. Fujino, A. Andoh, S. Bamba et al., "Increased expression of interleukin 17 in inflammatory bowel disease," Gut, vol. 52, no. 1, pp. 65-70, 2003.

[33] A. Globig, N. Hennecke, B. Martin et al., "Comprehensive intestinal $\mathrm{T}$ helper cell profiling reveals specific accumulation of IFN-gamma+IL-17+coproducing $\mathrm{CD}^{+}{ }^{+} \mathrm{T}$ cells in active inflammatory bowel disease," Inflammatory Bowel Diseases, vol. 20, no. 12, pp. 2321-2329, 2014.

[34] T. T. Pizarro, L. Pastorelli, G. Bamias et al., "SAMP1/YitFc mouse strain: a spontaneous model of Crohn's disease-like ileitis," Inflammatory Bowel Diseases, vol. 17, no. 12, pp. 2566-2584, 2011.

[35] Z. Hovhannisyan, J. Treatman, D. R. Littman, and L. Mayer, "Characterization of interleukin-17-producing regulatory $\mathrm{T}$ cells in inflamed intestinal mucosa from patients with inflammatory bowel diseases," Gastroenterology, vol. 140, no. 3, pp. 957-965, 2011.

[36] A. Ueno, H. Jijon, R. Chan et al., "Increased prevalence of circulating novel IL-17 secreting Foxp3 expressing CD4+ T cells and defective suppressive function of circulating Foxp3+ regulatory cells support plasticity between Th17 and regulatory $\mathrm{T}$ cells in inflammatory bowel disease patients," Inflammatory Bowel Diseases, vol. 19, no. 12, pp. 2522-2534, 2013.

[37] M. Sarra, I. Monteleone, C. Stolfi et al., "Interferon-gammaexpressing cells are a major source of interleukin-21 in inflammatory bowel diseases," Inflammatory Bowel Diseases, vol. 16, no. 8, pp. 1332-1339, 2010.

[38] D. Zehn and M. J. Bevan, "T cells with low avidity for a tissue-restricted antigen routinely evade central and peripheral tolerance and cause autoimmunity," Immunity, vol. 25, no. 2, pp. 261-270, 2006.

[39] N. T. Funderburg, S. R. Stubblefield Park, H. C. Sung et al., "Circulating $\mathrm{CD}^{+}$and $\mathrm{CD}^{+} \mathrm{T}$ cells are activated in inflammatory bowel disease and are associated with plasma markers of inflammation," Immunology, vol. 140, no. 1, pp. 8797, 2013.

[40] H. Cheroutre, "In IBD eight can come before four," Gastroenterology, vol. 131, no. 2, pp. 667-670, 2006.

[41] S. Nancey, S. Holvöet, I. Graber et al., "CD8 ${ }^{+}$cytotoxic T cells induce relapsing colitis in normal mice," Gastroenterology, vol. 131, no. 2, pp. 485-496, 2006.

[42] F. Hacini-Rachinel, S. Nancey, G. Boschetti et al., "CD4 ${ }^{+}$T cells and Lactobacillus casei control relapsing colitis mediated by $\mathrm{CD}^{+}$T cells," Journal of Immunology, vol. 183, no. 9, pp. 54775486, 2009.

[43] J. Qin, R. Li, J. Raes et al., "A human gut microbial gene catalogue established by metagenomic sequencing," Nature, vol. 464, no. 7285, pp. 59-65, 2010. 
[44] J. Li, H. Jia, X. Cai et al., "An integrated catalog of reference genes in the human gut microbiome," Nature Biotechnology, vol. 32, pp. 834-841, 2014.

[45] F. Fava and S. Danese, "Intestinal microbiota in inflammatory bowel disease: friend of foe?" World Journal of Gastroenterology, vol. 17, no. 5, pp. 557-566, 2011.

[46] S. J. Ott, M. Musfeldt, D. F. Wenderoth et al., "Reduction in diversity of the colonic mucosa associated bacterial microflora in patients with active inflammatory bowel disease," Gut, vol. 53, no. 5, pp. 685-693, 2004.

[47] H. Sokol and P. Seksik, "The intestinal microbiota in inflammatory bowel diseases: time to connect with the host," Current Opinion in Gastroenterology, vol. 26, no. 4, pp. 327-331, 2010.

[48] R. K. Sellon, S. Tonkonogy, M. Schultz et al., "Resident enteric bacteria are necessary for development of spontaneous colitis and immune system activation in interleukin-10-deficient mice," Infection and Immunity, vol. 66, no. 11, pp. 5224-5231, 1998.

[49] J. L. Round and S. K. Mazmanian, "The gut microbiota shapes intestinal immune responses during health and disease," Nature Reviews Immunology, vol. 9, no. 5, pp. 313-323, 2009.

[50] K. Smith, K. D. McCoy, and A. J. Macpherson, "Use of axenic animals in studying the adaptation of mammals to their commensal intestinal microbiota," Seminars in Immunology, vol. 19, no. 2, pp. 59-69, 2007.

[51] H. Chung, S. J. Pamp, J. A. Hill et al., "Gut immune maturation depends on colonization with a host-specific microbiota," Cell, vol. 149, no. 7, pp. 1578-1593, 2012.

[52] T. W. Hand, L. M. dos Santos, N. Bouladoux et al., "Acute gastrointestinal infection induces long-lived microbiota-specific $\mathrm{T}$ cell responses," Science, vol. 337, no. 6101, pp. 1553-1556, 2012.

[53] R. Duchmann, E. May, M. Heike, P. Knolle, M. Neurath, and K.-H. Meyer Zum Büschenfelde, "T cell specificity and cross reactivity towards enterobacteria, Bacteroides, Bifidobacterium, and antigens from resident intestinal flora in humans," Gut, vol. 44, no. 6, pp. 812-818, 1999.

[54] Y. Cong, S. L. Brandwein, R. P. McCabe et al., "CD4 ${ }^{+} \mathrm{T}$ cells reactive to enteric bacterial antigens in spontaneously colitic $\mathrm{C} 3 \mathrm{H} / \mathrm{HeJBir}$ mice: increased T helper cell type 1 response and ability to transfer disease," Journal of Experimental Medicine, vol. 187, no. 6, pp. 855-864, 1998.

[55] Q. Wang, R. M. McLoughlin, B. A. Cobb et al., "A bacterial carbohydrate links innate and adaptive responses through Tolllike receptor 2," The Journal of Experimental Medicine, vol. 203, no. 13, pp. 2853-2863, 2006.

[56] I. I. Ivanov, R. D. L. Frutos, N. Manel et al., "Specific microbiota direct the differentiation of IL-17-producing T-helper cells in the mucosa of the small intestine," Cell Host and Microbe, vol. 4, no. 4, pp. 337-349, 2008.

[57] K. Atarashi, J. Nishimura, T. Shima et al., "ATP drives lamina propria $\mathrm{T}_{H} 17$ cell differentiation," Nature, vol. 455, no. 7214, pp. 808-812, 2008.

[58] J. Duan, H. Chung, E. Troy, and D. L. Kasper, "Microbial colonization drives expansion of IL-1 receptor 1-expressing and IL-17-producing $\gamma / \delta$ T cells," Cell Host and Microbe, vol. 7, no. 2, pp. 140-150, 2010.

[59] V. Gaboriau-Routhiau, S. Rakotobe, E. Lecuyer et al., "The key role of segmented filamentous bacteria in the coordinated maturation of gut helper T cell responses," Immunity, vol. 31, no. 4, pp. 677-689, 2009.
[60] I. I. Ivanov, K. Atarashi, N. Manel et al., "Induction of intestinal Th17 cells by segmented filamentous bacteria," Cell, vol. 139, no. 3, pp. 485-498, 2009.

[61] M. Caselli, D. Tosini, R. Gafà, A. Gasbarrini, and G. Lanza, "Segmented filamentous bacteria-like organisms in histological slides of ileo-cecal valves in patients with ulcerative colitis," American Journal of Gastroenterology, vol. 108, no. 5, pp. 860861, 2013.

[62] I. Mosconi, M. B. Geuking, M. M. Zaiss et al., "Intestinal bacteria induce TSLP to promote mutualistic T-cell responses," Mucosal Immunology, vol. 6, no. 6, pp. 1157-1167, 2013.

[63] J. A. Hall, N. Bouladoux, C. M. Sun et al., "Commensal DNA limits regulatory $\mathrm{T}$ cell conversion and is a natural adjuvant of intestinal immune responses," Immunity, vol. 29, no. 4, pp. 637649, 2008.

[64] F. A. Carvalho, O. Koren, J. K. Goodrich et al., "Transient inability to manage proteobacteria promotes chronic gut inflammation in TLR5-deficient mice," Cell Host and Microbe, vol. 12, no. 2, pp. 139-152, 2012.

[65] M. A. Kinnebrew, C. G. Buffie, G. E. Diehl et al., "Interleukin 23 production by intestinal $\mathrm{CD}_{103}{ }^{+} \mathrm{CD}_{11 b^{+}}$dendritic cells in response to bacterial flagellin enhances mucosal innate immune defense," Immunity, vol. 36, no. 2, pp. 276-287, 2012.

[66] A. Izcue and F. Powrie, "Special regulatory T-cell review: regulatory $\mathrm{T}$ cells and the intestinal tract-patrolling the frontier," Immunology, vol. 123, no. 1, pp. 6-10, 2008.

[67] M. J. Lodes, Y. Cong, C. O. Elson et al., "Bacterial flagellin is a dominant antigen in Crohn disease," The Journal of Clinical Investigation, vol. 113, no. 9, pp. 1296-1306, 2004.

[68] B. Min, A. Thornton, S. M. Caucheteux et al., "Gut flora antigens are not important in the maintenance of regulatory T cell heterogeneity and homeostasis," European Journal of Immunology, vol. 37, no. 7, pp. 1916-1923, 2007.

[69] M. B. Geuking, J. Cahenzi, M. A. Lawson et al., "Intestinal bacterial colonization induced mutualistic regulatory $\mathrm{T}$ cell responses," Immunity, vol. 34, no. 5, pp. 794-806, 2011.

[70] S. K. Lathrop, S. M. Bloom, S. M. Rao et al., "Peripheral education of the immune system by colonic commensal microbiota," Nature, vol. 478, no. 7368, pp. 250-254, 2011.

[71] J. L. Round, S. M. Lee, J. Li et al., “The Toll-like receptor 2 pathway establishes colonization by a commensal of the human microbiota," Science, vol. 332, no. 6032, pp. 974-977, 2011.

[72] J. L. Round and S. K. Mazmanian, "Inducible Foxp3+ regulatory T-cell development by a commensal bacterium of the intestinal microbiota," Proceedings of the National Academy of Sciences of the United States of America, vol. 107, no. 27, pp. 12204-12209, 2010.

[73] K. Atarashi, T. Tanoue, T. Shima et al., "Induction of colonic regulatory T cells by indigenous Clostridium species," Science, vol. 331, no. 6015, pp. 337-341, 2011.

[74] K. Atarashi, T. Tanoue, K. Oshima et al., "Treg induction by a rationally selected mixture of Clostridia strains from the human microbiota," Nature, vol. 500, no. 7461, pp. 232-236, 2013.

[75] P. M. Smith, M. R. Howitt, N. Panikov et al., “The microbial metabolites, short-chain fatty acids, regulate colonic Treg cell homeostasis," Science, vol. 341, no. 6145, pp. 569-573, 2013.

[76] Y. Furusawa, Y. Obata, S. Fukuda et al., "Commensal microbederived butyrate induces the differentiation of colonic regulatory T cells," Nature, vol. 504, no. 7480, pp. 446-450, 2013.

[77] N. Singh, A. Gurav, S. Sivaprakasam et al., "Activation of Gpr109a, receptor for niacin and the commensal metabolite 
butyrate, suppresses colonic inflammation and carcinogenesis," Immunity, vol. 40, no. 1, pp. 128-139, 2014.

[78] R. Mariman, B. Kremer, M. van Erk, T. Lagerweij, F. Koning, and L. Nagelkerken, "Gene expression profiling identifies mechanisms of protection to recurrent trinitrobenzene sulfonic acid colitis mediated by probiotics," Inflammatory Bowel Diseases, vol. 18, no. 8, pp. 1424-1433, 2012.

[79] J. M. Uronis, J. C. Arthur, T. Keku et al., "Gut microbial diversity is reduced by the probiotic VSL\#3 and correlates with decreased TNBS-induced colitis," Inflammatory Bowel Diseases, vol. 17, no. 1, pp. 289-297, 2011.

[80] C. Di Giacinto, M. Marinaro, M. Sanchez, W. Strober, and M. Boirivant, "Probiotics ameliorate recurrent Thl-mediated murine colitis by inducing IL-10 and IL-10-dependent TGF- $\beta$ bearing regulatory cells," The Journal of Immunology, vol. 174, no. 6, pp. 3237-3246, 2005.

[81] C. O’Mahony, P. Scully, D. O’Mahony et al., “Commensalinduced regulatory $\mathrm{T}$ cells mediate protection against pathogenstimulated NF- $\kappa \mathrm{B}$ activation," PLoS Pathogens, vol. 4, no. 8, Article ID e1000112, 2008.

[82] Y. Cao, J. Shen, and Z. H. Ran, "Association between Faecalibacterium prausnitzii reduction and inflammatory bowel disease: a meta-analysis and systematic review of the literature," Gastroenterology Research and Practice, vol. 2014, Article ID 872725, 7 pages, 2014.

[83] X. Qiu, M. Zhang, X. Yang, N. Hong, and C. Yu, "Faecalibacterium prausnitzii upregulates regulatory $\mathrm{T}$ cells and anti-inflammatory cytokines in treating TNBS-induced colitis," Journal of Crohn's and Colitis, vol. 7, no. 11, pp. e558-e568, 2013.

[84] H. Zhang, J. B. Sparks, S. V. Karyala, R. Settlage, and X. M. Luo, "Host adaptive immunity alters gut microbiota," The ISME Journal, vol. 9, no. 3, pp. 770-781, 2014.

[85] K. Suzuki and S. Fagarasan, "How host-bacterial interactions lead to IgA synthesis in the gut," Trends in Immunology, vol. 29, no. 11, pp. 523-531, 2008.

[86] S. Brugman, K. Schneeberger, M. Witte et al., "T lymphocytes control microbial composition by regulating the abundance of Vibrio in the zebrafish gut," Gut Microbes, vol. 5, no. 6, pp. 737747, 2015.

[87] W. S. Garrett, G. M. Lord, S. Punit et al., "Communicable ulcerative colitis induced by T-bet deficiency in the innate immune system," Cell, vol. 131, no. 1, pp. 33-45, 2007.

[88] S. Kawamoto, M. Maruya, L. Kato et al., "Foxp3 $3^{+}$T cells regulate immunoglobulin a selection and facilitate diversification of bacterial species responsible for immune homeostasis," Immunity, vol. 41, no. 1, pp. 152-165, 2014.

[89] T. Feng, L. Wang, T. R. Schoeb, C. O. Elson, and Y. Cong, "Microbiota innate stimulation is a prerequisite for T cell spontaneous proliferation and induction of experimental colitis," The Journal of Experimental Medicine, vol. 207, pp. 1321-1332, 2010.

[90] S. Dasgupta and D. L. Kasper, "Relevance of commensal microbiota in the treatment and prevention of inflammatory bowel disease," Inflammatory Bowel Diseases, vol. 19, no. 11, pp. 2478-2489, 2013. 


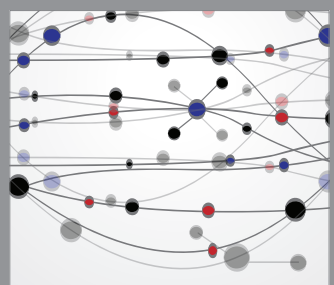

The Scientific World Journal
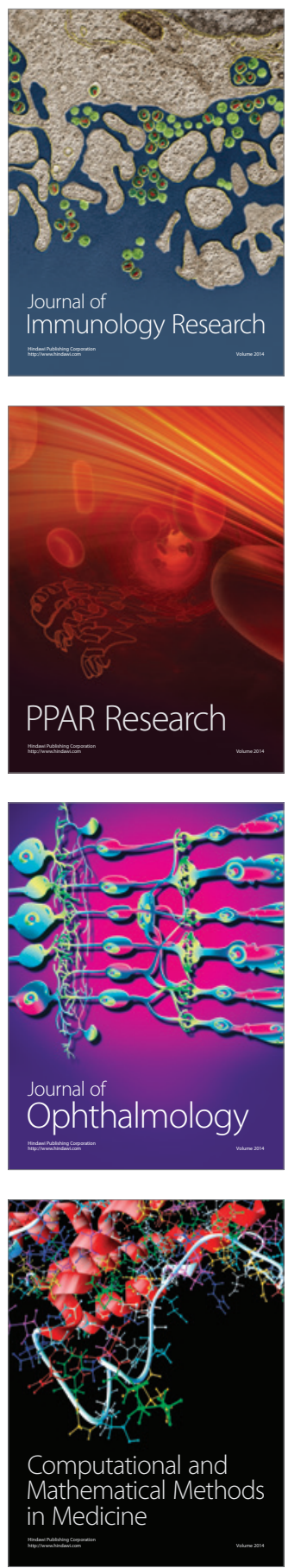

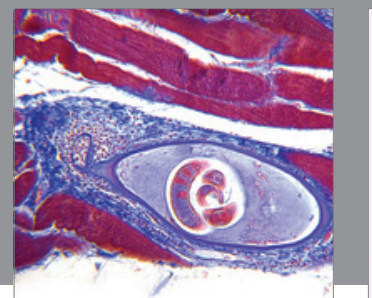

Gastroenterology

Research and Practice
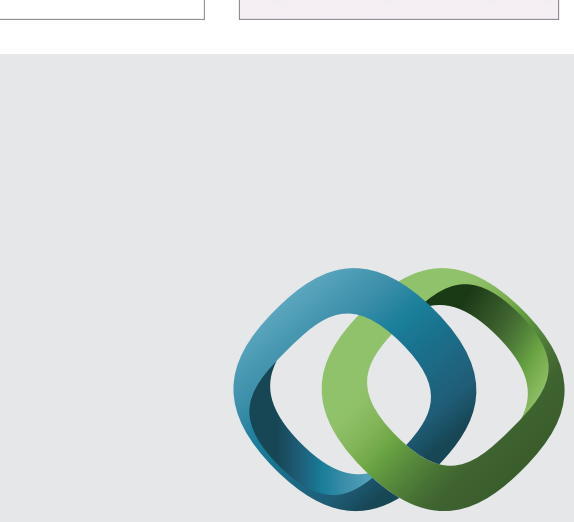

\section{Hindawi}

Submit your manuscripts at

http://www.hindawi.com
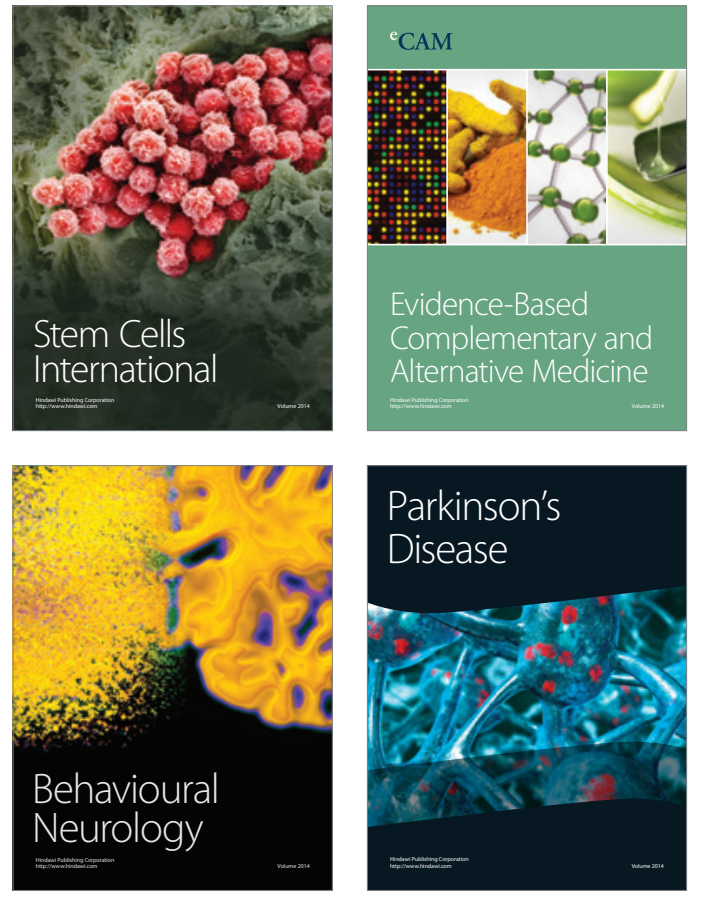
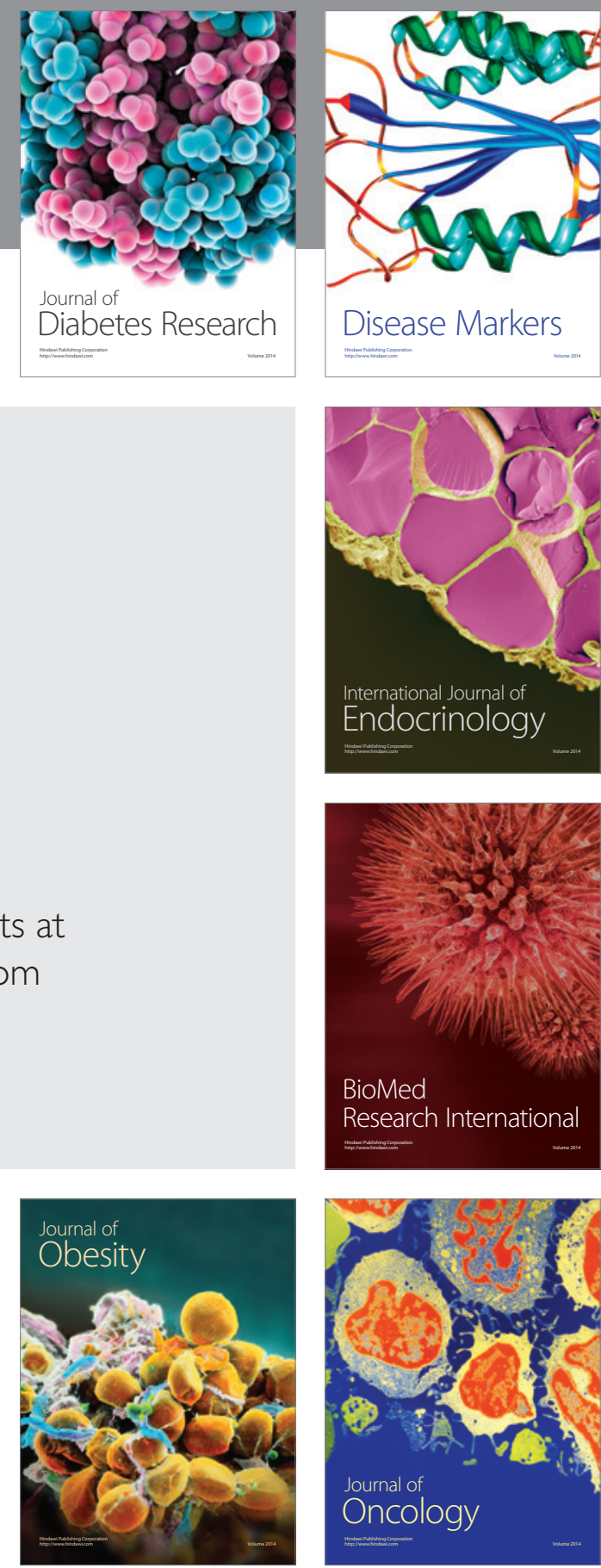

Disease Markers
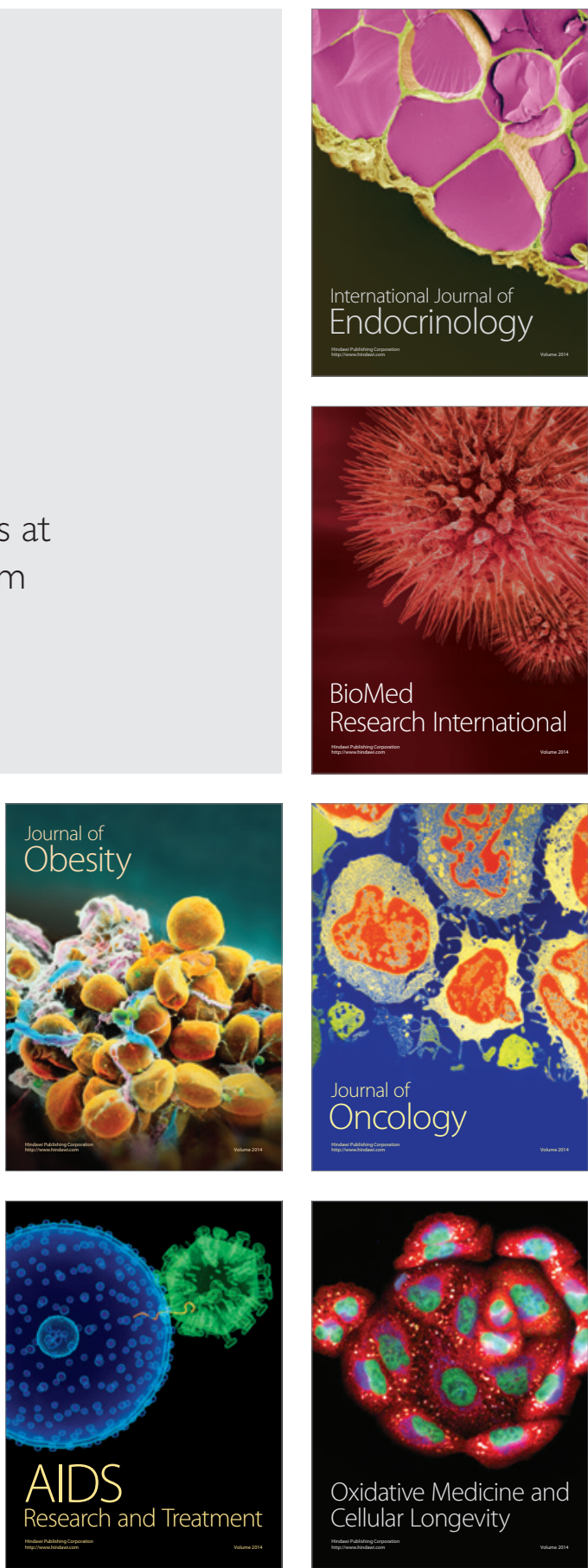